\title{
Microbial production of ectoine and hydroxyectoine as high-value chemicals
}

\author{
Mengshuang Liu' ${ }^{1,3}$, Hui Liư ${ }^{2}$, Meng Shi ${ }^{4}$, Mingyue Jiang ${ }^{1,3}$, Lingling Li ${ }^{1}$ and Yanning Zheng ${ }^{1 *}$ (D)
}

\begin{abstract}
Ectoine and hydroxyectoine as typical representatives of compatible solutes are not only essential for extremophiles to survive in extreme environments, but also widely used in cosmetic and medical industries. Ectoine was traditionally produced by Halomonas elongata through a "bacterial milking" process, of which the marked feature is using a highsalt medium to stimulate ectoine biosynthesis and then excreting ectoine into a low-salt medium by osmotic shock. The optimal hydroxyectoine production was achieved by optimizing the fermentation process of Halomonas salina. However, high-salinity broth exacerbates the corrosion to fermenters, and more importantly, brings a big challenge to the subsequent wastewater treatment. Therefore, increasing attention has been paid to reducing the salinity of the fermentation broth but without a sacrifice of ectoine/hydroxyectoine production. With the fast development of functional genomics and synthetic biology, quite a lot of progress on the bioproduction of ectoine/hydroxyectoine has been achieved in recent years. The importation and expression of an ectoine producing pathway in a non-halophilic chassis has so far achieved the highest titer of ectoine $(\sim 65 \mathrm{~g} / \mathrm{L})$, while rational flux-tuning of halophilic chassis represents a promising strategy for the next-generation of ectoine industrial production. However, efficient conversion of ectoine to hydroxyectoine, which could benefit from a clearer understanding of the ectoine hydroxylase, is still a challenge to date.
\end{abstract}

Keywords: Metabolic engineering, Halophiles, Model microorganisms, Ectoine, Hydroxyectoine

\section{Background}

Increased environmental osmolarity will trigger the outflow of intracellular water across the cell membrane, resulting in the loss of turgor and then the shrinkage of cell. The overcrowding of biomacromolecules lowers their diffusion rates in cytoplasm, affects normal cellular physiological activities, and ultimately threatens cell survival $[1,2]$. To avoid the detrimental effects caused by the high environmental osmolarity, some halophilic microorganisms are able to balance the intra- and extracellular osmotic pressure by accumulating large amounts of specific small organic molecules called compatible

\footnotetext{
*Correspondence: zhengyn@im.ac.cn

${ }^{1}$ State Key Laboratory of Microbial Resources, Institute of Microbiology,

Chinese Academy of Sciences, No. 1 Beichen West Road, Chaoyang District, Beijing 100101, China

Full list of author information is available at the end of the article
}

solutes, whose massive accumulation does not disturb normal cellular process $[3,4]$. Compatible solutes, mainly including sugars (sucrose, trehalose and so on), polyols (glycerol, glucosyl-glycerol, mannosyl-glycerol, arabitol, sorbitol, mannitol and so on), amino acids and their derivatives (proline, glycine betaine, ectoine, hydroxyectoine and so on), are widely distributed in nature $[5,6]$.

As typical representatives of compatible solutes, ectoine (1,4,5,6-tetrahydro-2-methyl-4-pyrimidinecarboxylic acid) is a cyclic derivative of aspartate while hydroxyectoine (1,4,5,6-tetrahydro-2-methyl-5-hydroxy4-pyrimidinecarboxylic acid) is a hydroxylated derivative of ectoine [7, 8]. In addition to acting as osmotic protective agents, ectoine and hydroxyectoine are also excellent biofunctional stabilizers, skin protectors and potential drugs for diseases, such as Alzheimer's and rhinoconjunctivitis symptom [9-14]. Ectoine has become a

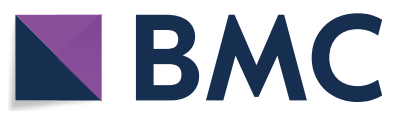

(c) The Author(s) 2021. This article is licensed under a Creative Commons Attribution 4.0 International License, which permits use, sharing, adaptation, distribution and reproduction in any medium or format, as long as you give appropriate credit to the original author(s) and the source, provide a link to the Creative Commons licence, and indicate if changes were made. The images or other third party material in this article are included in the article's Creative Commons licence, unless indicated otherwise in a credit line to the material. If material is not included in the article's Creative Commons licence and your intended use is not permitted by statutory regulation or exceeds the permitted use, you will need to obtain permission directly from the copyright holder. To view a copy of this licence, visit http://creativeco mmons.org/licenses/by/4.0/. The Creative Commons Public Domain Dedication waiver (http://creativecommons.org/publicdomain/ zero/1.0/) applies to the data made available in this article, unless otherwise stated in a credit line to the data. 
high-demand product because of its wide application in biotechnology, cosmetics and medicine, and also represents a multibillion-dollar market, with an annual market demanding of about 15,000 tons and a retail price of about $1000 \mathrm{USD} / \mathrm{kg}$ [15]. The current process for hydroxyectoine bioproduction can only provide a mixture of ectoine and hydroxyectoine, which are chemically similar compounds. Given it is not easy to isolate hydroxyectoine from the mixture of ectoine and hydroxyectoine, bioproduction of hydroxyectoine on a large scale was economically unattractive. However, hydroxyectoine has a higher glass transition temperature than ectoine, making it be a better desiccation protectant $[16,17]$. Therefore, increasing attention has been paid to the microbial production of ectoine/hydroxyectoine in recent years.

The biosynthesis of ectoine from aspartate is carried out by sequential catalysis of five enzymes, namely, L-aspartate kinase (Ask), L-aspartate- $\beta$-semialdehyde dehydrogenase (Asd), L-2,4-diaminobutyrate aminotransferase (EctB), L-2,4-diaminobutyrate acetyltransferase (EctA) and ectoine synthase (EctC) $[18,19]$. Ectoine hydroxylase (EctD), which catalyzes the conversion of ectoine to hydroxyectoine through a regio- and stereo-specific hydroxylation reaction, has also been found in many native ectoine producers $[8,20,21]$. Genes encoding EctB, EctA and EctC are usually present as a gene cluster (ectABC), sometimes with ectD in the context [17]. The expression of $\operatorname{ect} A B C(D)$ is triggered by an increase of environmental salinity [22]. Disruption of $\operatorname{ect} A B C(D)$ will make halophiles sensitive to high-salinity levels, indicating that ectoine and hydroxyectoine play important roles for microorganisms in adaptation to high-salinity environment $[17,23]$. In addition to being compatible solutes, ectoine and hydroxyectoine are able to be degraded by some microorganisms for carbon and nitrogen sources [24, 25]. Based on the genome analysis of H. elongata DSM 2581T, Schwibbert et al. described the ectoine degradation pathway for the first time [26]. Their proposed Doe pathway converts ectoine back to aspartate with a different set of enzymes, namely, ectoine hydrolase (DoeA), $\quad N$ - $\alpha$-acetyl-L-2,4-diaminobutyric acid deacetylase (DoeB), diaminobutyric acid transaminase (DoeD) and aspartate-semialdehyde dehydrogenase (DoeC) (Fig. 1). The pathway for the catabolism of hydroxyectoine has been proposed in Ruegeria pomeroyi. EutA, EutB and EutC are probably responsible for the conversion of hydroxyectoine into ectoine, which is further degraded into aspartate using the above-mentioned Doe pathway [27]. However, Christopher et al. found that the EutD (DoeA)/EutE (DoeB) from $R$. pomeroyi could degrade hydroxyectoine into hydroxy-L-2,4-diaminobutyrate (hydroxy-DABA) via hydroxy- $N \alpha$-acetylL-2,4-diaminobutyrate (hydroxy-N $\alpha$-ADABA) as an

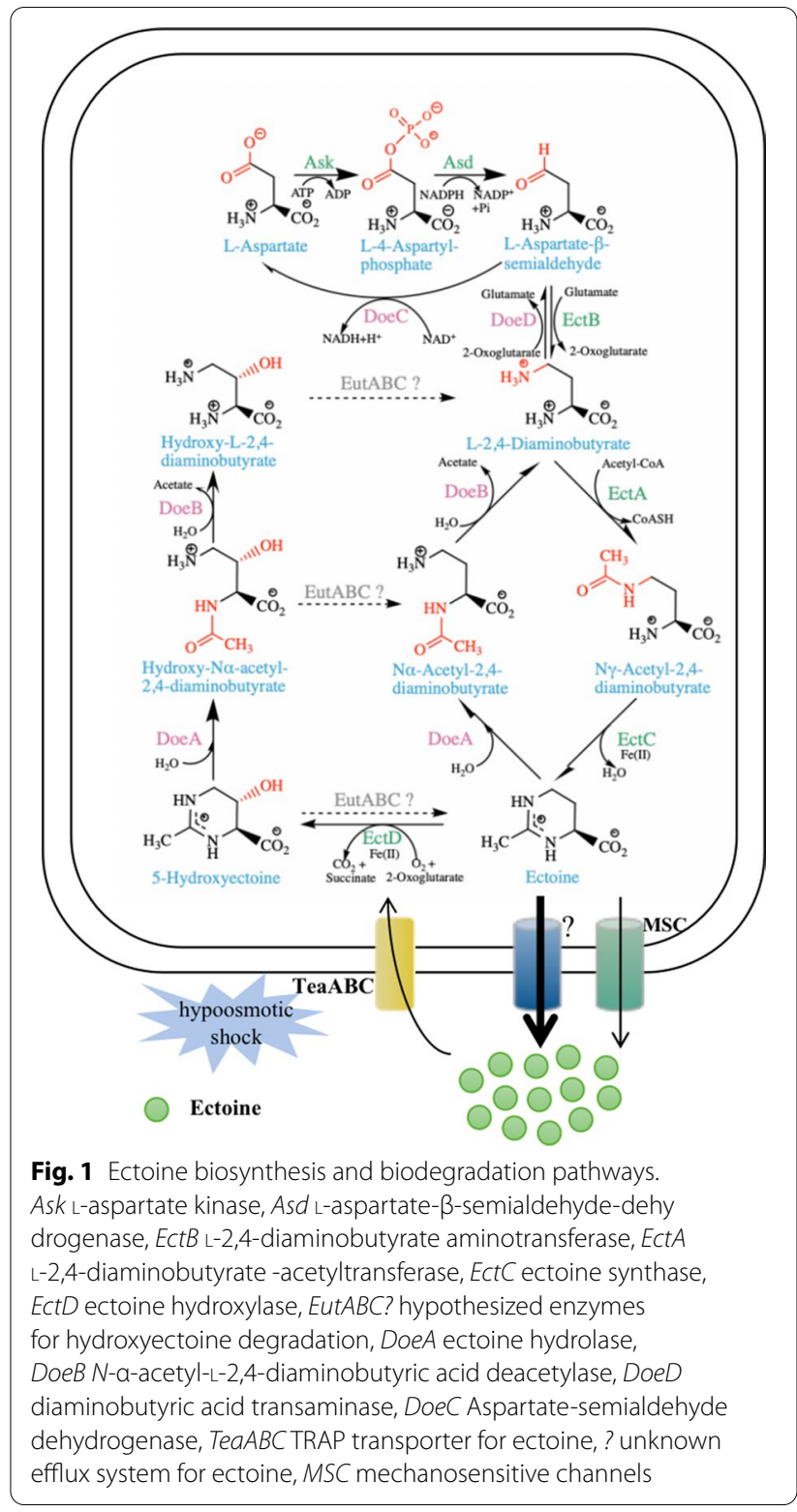

intermediate [28], implying that a different pathway for hydroxyectoine degradation may exist (Fig. 1).

Industrially, ectoine is produced by Halomonas elongata through a "bacterial milking" process [29]. In this process, high-salt medium is used to stimulate ectoine biosynthesis, and then the bacterial cells are shocked by low-salt medium to rapidly release the synthesized ectoine into the medium. Hydroxyectoine production can be improved by increasing medium salinity and culture temperature [30]. However, high-salt medium exacerbates the corrosion of conventional steel fermenter and increases the difficulty of wastewater treatment [31-33], all of which brings extra cost of ectoine/ 
hydroxyectoine bioproduction. Given the disadvantages caused by high-salt medium, researchers have made efforts to lower the salinity normally required for efficient biosynthesis of ectoine/hydroxyectoine (Table 1).

Synthetic biology, through which the gene regulation can be eliminated to artificially control the gene expression, plays a key role in the development of high-value chemicals, including ectoine/hydroxyectoine. Quite a lot of advances in improving ectoine/ hydroxyectoine production under low-salinity conditions and in enhancing the exportation of ectoine/ hydroxyectoine have been achieved through the synthetic biology. Given that E. coli and Corynebacterium glutamicum normally grow at low-salinity levels, they have recently been used as bacterial chassis for ectoine/ hydroxyectoine production [34,35]. Though E. coli and C. glutamicum do not naturally accumulate ectoine and hydroxyectoine, they can be endowed with the ability to produce ectoine/hydroxyectoine through synthetic biology. More recently, enhanced ectoine production has been achieved by rational flux-tuning in a chromosomally engineered $H$. bluephagenesis, which allows open unsterile and continuous growth conditions, providing a promising bacterial chassis for next generation of industrial biotechnology [36].

\section{Microbial production of ectoine/hydroxyectoine in halophilic bacteria Halomonas elongata}

Ectoine was initially produced by the "bacterial milking" process in a non-engineered halophilic bacterium H. elongata. By adopting the "bacterial milking" process, a final ectoine titer of $7.4 \mathrm{~g} / \mathrm{L}$ was achieved after repeatedly performing this process at least nine times, with a productivity of $0.22 \mathrm{~g} / \mathrm{L} / \mathrm{h}$. Mechanosensitive channels (MSC), which are responsible for the excretion of compatible solutes in many halophilic microorganisms when suffering hypoosmotic shock, only make a small contribution to the ectoine excretion in $H$. elongata $[37,38]$. Therefore, $H$. elongata should have an efflux system either as a universal compatible solute transporter or as a specific ectoine exporter [38]. By deleting the Trap-TeaABC transporter for ectoine uptake and disrupting the Doe pathway for ectoine degradation, Kunte's research group developed a "super-leaky" H. elongata mutant, which is capable of exporting synthesized ectoine to the medium without hypoosmotic shock [26, 29, 39]. The above-mentioned techniques make an annual production of ectoine in tons possible, but high-salt media are still required.

When $H$. elongata was grown in a synthetic medium containing $4.27 \mathrm{M} \mathrm{NaCl}$ at $40{ }^{\circ} \mathrm{C}$, it produced more

Table 1 Microbial production of ectoine and hydroxyectoine

\begin{tabular}{|c|c|c|c|c|c|c|}
\hline Strain & Salinity $^{\mathrm{a}}(\mathrm{M})$ & Titer $(g / L)$ & $\begin{array}{l}\text { Productivity } \\
(\mathrm{g} / \mathrm{L} / \mathrm{h})\end{array}$ & Yield (g/g) & Carbon source & References \\
\hline \multicolumn{7}{|l|}{ Ectoine } \\
\hline H. elongata DSM142 & 2.57 & 7.4 & 0.22 & 0.11 & Glucose & {$[30]$} \\
\hline B. epidermis DSM 20659 & 1.42 & 8 & 0.08 & 0.05 & Monosodium glutamate & {$[31]$} \\
\hline H. salina DSM5928 & 1.81 & 14.86 & 0.32 & 0.14 & Monosodium glutamate & {$[33]$} \\
\hline H. salina BCRC17875 & 2.47 & 13.96 & 0.29 & NA & Monosodium glutamate & {$[42]$} \\
\hline H. hydrothermalis Y2 & 1.14 & 10.5 & 0.22 & $0.21^{b}$ & Monosodium glutamate and glucose & {$[46]$} \\
\hline E. coli DH5a (pASK-ectABC) & 0.08 & 6 & 0.04 & NA & Glucose & {$[32]$} \\
\hline E. coli BW25113 & 0.4 & 25.1 & 1.04 & NA & Glycerol and aspartate & {$[51]$} \\
\hline E. coli ECT05 & 0.03 & 25.1 & 0.84 & 0.11 & Glucose & {$[34]$} \\
\hline E. coli DH5a (pASK_ectABCD $\left.{ }_{m}\right)$ & 0.22 & 1.7 & NA & 0.36 & Glycerol & {$[59]$} \\
\hline C. glutamicum Ect-2 & 0.03 & 4.5 & 0.28 & NA & Glucose & {$[66]$} \\
\hline C. glutamicum Ecto5 & 0.01 & 22 & 0.32 & 0.16 & Glucose & {$[68]$} \\
\hline C. glutamicum ect $A B C^{\text {opt }}$ & 0.03 & 65 & 1.16 & $0.19^{c}$ & Glucose and molasses & {$[35]$} \\
\hline \multicolumn{7}{|l|}{ Hydroxyectoine } \\
\hline H. salina BCRC17875 & 0.56 & 2.9 & NA & NA & Monosodium glutamate & {$[43]$} \\
\hline H. polymorpha ALU3/EctBACD & 0 & 2.8 & 0.02 & NA & MeOH and sorbitol & {$[72]$} \\
\hline E. coli FF4169 (pMP41) & 0.4 & 2.13 & 0.09 & NA & Glucose and ectoine & {$[57]$} \\
\hline E. coli DH5a (pASK_ectABCDask) & 0.26 & 1.6 & NA & 0.34 & Glycerol & {$[59]$} \\
\hline
\end{tabular}

NA not available

a The salinity is shown as the combined concentration of sodium and potassium salts in the medium

b The yield here is calculated with monosodium glutamate

c The yield here is calculated with glucose and molasses 
hydroxyectoine than ectoine. However, it is economically infeasible to isolate hydroxyectoine from the mixture of ectoine and hydroxyectoine because of their high chemical similarity, making the industrial production of hydroxyectoine unattractive $[6,9]$. Therefore, $H$. elongata was engineered to improve the conversion of ectoine to hydroxyectoine by heterogenous expression of the ectoine hydroxylase gene $(t h p D)$ from Streptomyces chrysomallus, the engineered strain was capable of converting all ectoine to hydroxyectoine [40]. But unfortunately, the medium salinity used in the study is still high (1.7 M).

\section{Brevibacterium epidermis}

Brevibacterium epidermis, which naturally produces ectoine, can tolerate salinities as high as $2 \mathrm{M} \mathrm{NaCl}$. Its ectoine production correlates positively with the $\mathrm{NaCl}$ concentrations up to $1 \mathrm{M}$. In addition, $B$. epidermis has no ectD gene, so it is unable to synthesize hydroxyectoine, which is a common by-product in ectoine biosynthesis [24]. B. epidermis has been used for ectoine production with a salt level of $1.42 \mathrm{M}$. The fed-batch fermentation of this bacterium using monosodium glutamate as substrate achieved an ectoine titer of $8 \mathrm{~g} / \mathrm{L}$, a productivity of $0.08 \mathrm{~g} / \mathrm{L} / \mathrm{h}$, and a yield of $0.05 \mathrm{~g} / \mathrm{g}$ monosodium glutamate [31]. B. epidermis has several advantages in ectoine production when compared with $H$. elongata. Firstly, it uses a lower salt level $(\sim 1.42 \mathrm{M})$ than H. elongata $(\sim 2.57 \mathrm{M} \mathrm{NaCl})$. Secondly, it can avoid a further consumption of ectoine and simplify the downstream ectoine recovery process, as it is naturally unable to make by-product hydroxyectoine. However, relatively high salinity and hypoosmotic shock are also required for efficient ectoine production by B. epidermis.

\section{Halomonas salina}

Halomonas salina DSM 5928 can synthesize and secrete ectoine at a constant extracellular osmotic pressure [41]. The $\mathrm{NaCl}$ concentrations ranging from $0.5 \mathrm{M}$ to $2 \mathrm{M}$ do not affect the final ectoine production, although a lower salt level contributes to a better excretion of ectoine. Therefore, $H$. salina exhibits advantages over $H$. elongata and B. epidermis in terms of medium-salt production of ectoine. When using monosodium glutamate as the carbon and nitrogen source in a batch fermentation, $H$. salina DSM 5928 produced $6.9 \mathrm{~g} / \mathrm{L}$ ectoine at a salt level of $0.5 \mathrm{M} \mathrm{NaCl}$, with a productivity of $0.33 \mathrm{~g} / \mathrm{L} / \mathrm{h}$ and a secretion rate of $61.6 \%$ [41]. To achieve a better ectoine production, Lang et al. adopted a two-phase strategy. They firstly grew bacterial cells in a batch fermentation, then harvested the cells, and finally used phosphate-limited non-growing cells for ectoine production. By separating cell growth from ectoine biosynthesis, $14.86 \mathrm{~g} / \mathrm{L}$ ectoine was produced by $H$. salina DSM 5928, with a productivity of $0.32 \mathrm{~g} / \mathrm{L} / \mathrm{h}$ and a yield of $0.14 \mathrm{~g} / \mathrm{g}$ monosodium glutamate. In addition, about $79 \%$ of ectoine was excreted into the medium [33].

Benefiting from the natural property of ectoine excretion, H. salina DSM 5928 can achieve a high ectoine production without hypoosmotic shock. Simultaneous ectoine biosynthesis and excretion not only simplifies the process of ectoine production, but also reduces the intracellular ectoine consumption by preventing ectoine from entering the Doe catabolic pathway. Additionally, dissolved oxygen is an important factor for efficient ectoine production. However, high cell density fermentation, which is always adopted for enhanced ectoine production by $H$. elongata and B. epidermis, can cause an undersupply of dissolved oxygen. An alternative strategy for reducing the consumption of oxygen is to use elementlimited non-growing cells. Indeed, two-fold more ectoine was produced by non-growing $H$. salina DSM 5928 cells when compared with its growing cells.

Halomonas salina BCRC 17875 has also been used for the production of ectoine/hydroxyectoine [42, 43]. By optimizing the agitation speed and medium composition, $13.96 \mathrm{~g} / \mathrm{L}$ ectoine was finally obtained using monosodium glutamate as substrate, with a productivity of $0.29 \mathrm{~g} / \mathrm{L} / \mathrm{h}$ [42]. In addition, $2.9 \mathrm{~g} / \mathrm{L}$ hydroxyectoine was achieved in medium supplemented with $50 \mathrm{mM} \alpha$-ketoglutarate and $1 \mathrm{mM}$ iron, representing the highest hydroxyectoine production to date [43].

\section{Halomonas hydrothermalis}

Halomonas hydrothermalis, originally isolated from the alkaline pulp mill wastewater, grows well at salt levels ranging from 0 to $3 \mathrm{M} \mathrm{NaCl}$ [44]. It expresses both ectoine biosynthesis pathway and ectoine degradation pathway, with the ectABC and doeABXCD gene clusters responsible for ectoine biosynthesis and ectoine degradation, respectively. Moreover, it also has an ectD gene locating remotely from the ectABC gene cluster [45]. By deleting the ectD and $d o e A$ genes, the ectoine production was increased from 5.5 to $7.2 \mathrm{~g} / \mathrm{L}$ in a batch fermentation [46]. By further knocking out the mrp gene, whose protein product is a $\mathrm{Na}^{+} / \mathrm{H}^{+}$antiporter and plays an important role in $\mathrm{pH}$ and osmotic homeostasis, the optimal NaCl concentration for ectoine biosynthesis dropped from 1.7 to $1.4 \mathrm{M}$ [47]. Finally, the engineered $H$. hydrothermalis $\mathrm{Y} 2 / \Delta e c t D / \Delta$ doeA/ $\Delta m r p$ grown in the fermentation broth supplemented with $\sim 1 \mathrm{M} \mathrm{NaCl}$ accumulated up to $10.5 \mathrm{~g} / \mathrm{L}$ of ectoine in a fed-batch fermentation, with a productivity of $0.22 \mathrm{~g} / \mathrm{L} / \mathrm{h}$, a yield of $0.21 \mathrm{~g} / \mathrm{g}$ monosodium glutamate and an ectoine excretion rate of $62 \%$.

Though the engineered strain $H$. hydrothermalis $\mathrm{Y} 2 / \Delta e c t D / \Delta$ doeA/ $m$ mrp produced less ectoine than $H$. salina, the salinity level required for maximal ectoine 
production can be further lowered by limiting the $\mathrm{Na}^{+}$ outflow, representing a new strategy for the low-salt production of compatible solutes. However, ectoine production decreased by $\sim 20 \%$ in the late stage of $H$. hydrothermalis $\mathrm{Y} 2 / \Delta e c t D / \Delta d o e A$ fermentation, indicating that $H$. hydrothermalis may still have some unknown ectoine degradation pathways. However, a further investigation is needed to verify this assumption. The TRAPTeaABC transporter is responsible for ectoine uptake, and the deletion of tea $\mathrm{C}$ and teaBC genes in $\mathrm{H}$. elongata can greatly enhance the excretion of ectoine, yielding a "super leaky" mutant. It suggests the function of TeaABC is probably to transport the extracellular ectoine into the cell and also participate in the negative regulation of ectoine biosynthesis [39]. Similar to H. elongata, the tea $A B C D$ gene cluster also exists in the genome of $H$. hydrothermalis [45]. Therefore, the deletion of teaABCD gene cluster is a promising strategy to further improve the production of ectoine in $H$. hydrothermalis.

\section{Microbial production of ectoine/hydroxyectoine in non-natural host strains \\ Escherichia coli}

Escherichia coli is widely used as host for heterologous protein expression due to its long history of laboratory culture, clear genetic background, ease of manipulation, and good compatibility with heterologous proteins [48]. In addition, $E$. coli does not have neither ectD gene nor doe $A B C D$ genes, making it unable to convert or degrade the synthesized ectoine [49]. Therefore, $E$. coli has been engineered for heterologous production of ectoine with low-salt medium. Louis et al. expressed the ect $A B C$ gene cluster from Marinococcus halophilus in E. coli XL1Blue for the production of ectoine, representing the first successful attempt of introducing ectoine biosynthetic pathway into $E$. coli [50]. The synthesized ectoine increased the tolerance of the engineered $E$. coli to the hyperosmotic stress (up to $0.85 \mathrm{M} \mathrm{NaCl}$ ). Subsequently, Schubert et al. introduced a tet promoter controlled ect$A B C$ from the halophilic bacterium Chromohalobacter salexigens into the E. coli DH5 $\alpha$ [32]. Under the control of the inducible tet promoter, the cell growth phase of $E$. coli was separated from its ectoine biosynthesis phase. About $6 \mathrm{~g} / \mathrm{L}$ ectoine was excreted by the engineered $E$. coli $\mathrm{DH} 5 \alpha$ using glucose as substrate, with an ectoine productivity of only $0.04 \mathrm{~g} / \mathrm{L} / \mathrm{h}$, which is much lower than that achieved in halophilic bacteria. Thus, ectoine production still need to be improved by employing inducible promoters with stronger and more stable expression of the ectoine biosynthetic pathway. An arabinoseinducible promoter was subsequently selected to drive the expression of $H$. elongata ect $A B C$ gene cluster in $E$. coli BW25113 [51]. The engineered E. coli achieved an ectoine titer of up to $25.1 \mathrm{~g} / \mathrm{L}$ and an ectoine productivity of $1.04 \mathrm{~g} / \mathrm{L} / \mathrm{h}$ by whole-cell catalysis when the bacterial cells were cultured with glycerol and aspartate as substrates. The whole-cell biocatalysis represents an alternative strategy to improve the ectoine bioproduction in engineered strain.

Escherichia coli W3110 has been successfully applied to synthesize aspartate-derived products, such as L-threonine and 3-aminopropionic acid, implying its potential in ectoine biosynthesis $[52,53]$. Ning et al. successfully introduced the ect $A B C$ gene cluster from $H$. elongata into E. coli W3110 and uncoupled the gene expression from osmotic pressure induction by using a strong trc promoter [34]. To further improve the ectoine production, they made efforts to provide the bacterial cells with sufficient precursor aspartate- $\beta$-semialdehyde (ASA) and then increase the metabolic flux from ASA to ectoine. Thus, the thrA gene coding for a bifunctional aspartate kinase/homoserine dehydrogenase was deleted to block the L-threonine branch, and the lys $C$ gene encoding the feedback inhibition resistant aspartokinase was overexpressed to complement the aspartokinase activity impaired by $t h r A$ deletion. The native promoter of $p p c$ gene for phosphoenolpyruvate carboxylase was also replaced with a stronger $t r c$ promoter, and the $i c l R$ gene, whose protein product is responsible for the transcriptional repression of the glyoxylate branch, was deleted to increase the supply of oxaloacetate. Finally, the engineered E. coli ECT05 accumulated $25.1 \mathrm{~g} / \mathrm{L}$ ectoine in a fed-batch fermentation using glucose as substrate, with a productivity of $0.84 \mathrm{~g} / \mathrm{L} / \mathrm{h}$ and a yield of $0.11 \mathrm{~g} / \mathrm{g}$ glucose. Altogether, a high ectoine production has been achieved at a salinity of as low as $0.03 \mathrm{M}$ through the metabolic engineering of $E$. coli (Fig. 2a).

Given that EctB is a rate-limiting enzyme for ectoine biosynthesis, Chen et al. established a high-throughput screening method in E. coli to screen $\mathrm{HeEctB}$ (ectB from $H$. elongata) variants with increased activities by engineering the regulatory protein $\mathrm{AraC}$ to use ectoine as its effector [54]. The expression of the quantitative reporter GFP is positively correlated with the ectoine production. Finally, a mutant (D180V/F320Y/Q325R) with obviously improved ectoine production was obtained by combining the directed evolution and high-throughput screening. More recently, the crystal structure of $C s E c t B$ (ectB from C. salexigens DSM 3043) has been determined. When structurally compared with the $C s E c t B$, all of the three point mutations (D180V/F320Y/Q325R) of $\mathrm{HeEctB}$ variant are located on the protein surface but not the active center [55]. These mutations may be responsible for the increased structural flexibility by affecting the electrostatic interaction (D180V), the hydrophobic patch (F320Y) and the surface charge alteration (Q325R) [55]. 

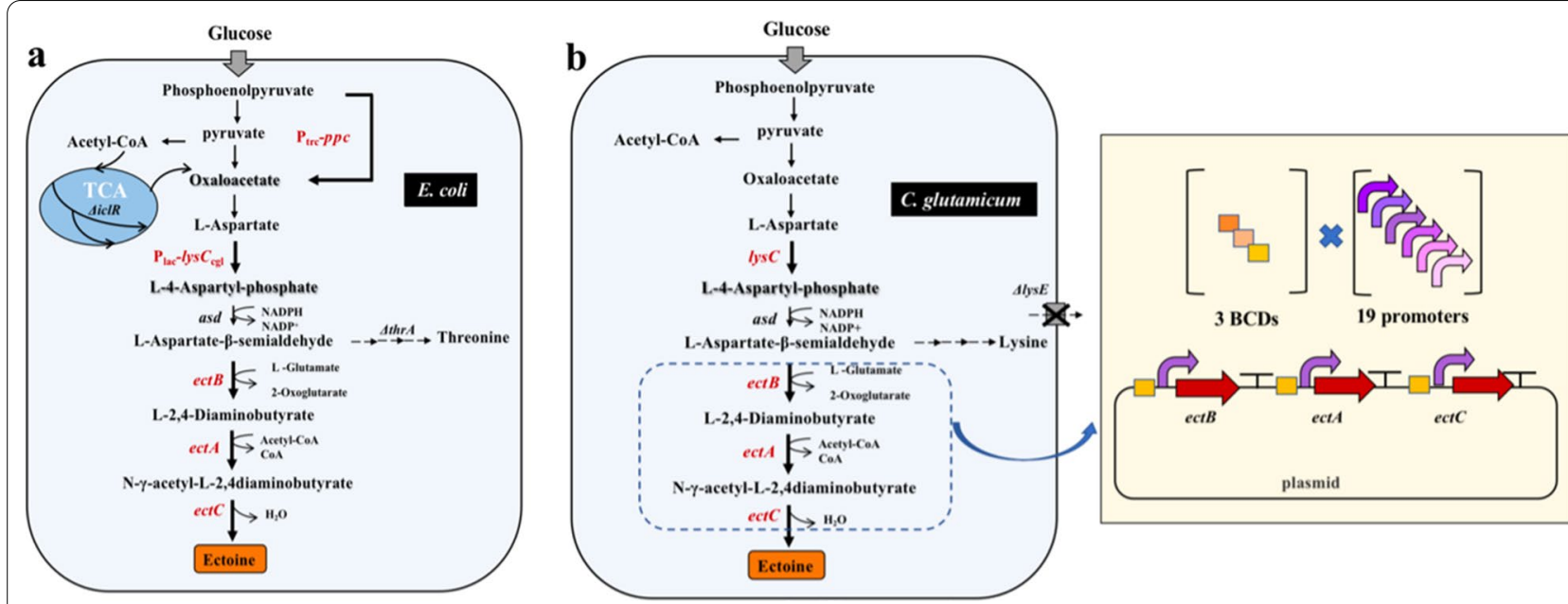

Fig. 2 Metabolic engineering modification strategy for ectoine production by E. coli and C. glutamicum. a Metabolic engineering modification strategy for ectoine production by E. coli. b Metabolic engineering modification strategy for ectoine production by C. glutamicum. Control element library for transcriptionally balanced expression of the ectoine synthesizes related genes. The library was constructed from 3 BCDs (bicistronic designed elements), 19 promoters and the terminator rrnBT1T2

The solved crystal structure of EctB will definitely contribute to the rational design of this rate-limiting enzyme for more efficient production of ectoine, though a good balance between its activity and stability should be taken into consideration.

Escherichia coli has also been used for the production of hydroxyectoine. Under the control of its salt-inducible native promoter, the whole ectABCD-ask gene cluster from P. stutzeri DSM5190 was successfully expressed in E. coli $\mathrm{DH} 5 \alpha$. The engineered strain requires a medium supplemented with $0.34 \mathrm{M} \mathrm{NaCl}$ for efficient hydroxyectoine production [56]. Subsequently, Czech et al. expressed ectD from P. stutzeri A1501 in E. coli FF4169 (defective in the trehalose synthesis) under the control of the inducible tet promoter. The added ectoine, which was imported into the cells with the help of osmotic stress inducible ProP and ProU transporters, was completely converted to hydroxyectoine in a shake-flask cultivation. Finally, a hydroxyectoine titer of $2.13 \mathrm{~g} / \mathrm{L}$ and a productivity of $0.09 \mathrm{~g} / \mathrm{L} / \mathrm{h}$ were obtained using a medium supplemented with 0.4 M salt [57]. However, it is still not economical to directly convert ectoine to hydroxyectoine in bulk because of the high price of ectoine.

In addition, the ectoine/hydroxyectoine biosynthetic gene clusters are usually obtained from halophilic microorganisms. When these gene clusters are expressed in $E$. coli, the activities of their gene products (enzymes) could be greatly impaired [58]. Therefore, Bethlehem et al. expressed the ectoine/hydroxyectoine biosynthetic genes from a non-halophilic bacterium (Acidiphilium cryptum) in E. coli [59]. Specifically, E. coli carrying pASK_ ectABCD ${ }_{m}$ and pASK_ectABCDask were used for the production of ectoine and hydroxyectoine, respectively. The pASK_ectABCD ${ }_{m}$ expresses EctABC and an inactivated EctD, while the pASK_ectABCDask expresses Ect$\mathrm{ABCD}$ and an additional aspartokinase. In a shake-flask cultivation using glycerol as substrate, an ectoine titer of $1.7 \mathrm{~g} / \mathrm{L}$ and a productivity of $0.36 \mathrm{~g} / \mathrm{g}$ glycerol were achieved with almost no detectable byproducts. In contrast to the ectoine production, a hydroxyectoine titer of $1.6 \mathrm{~g} / \mathrm{L}$ and a productivity of $0.34 \mathrm{~g} / \mathrm{g}$ glycerol were obtained and only $4 \%$ ectoine was detected as a byproduct, representing a promising strategy for the production of hydroxyectoine economically.

\section{Corynebacterium glutamicum}

Corynebacterium glutamicum, which has a long history in the production of amino acids, especially glutamate and lysine, is another microbial chassis commonly used [60-62]. The feedback inhibition resistant aspartokinase has been developed to provide lysine production with plenty of ASA precursor [63], which is also an important precursor compound for the biosynthesis of ectoine. Given the large amount of lysine produced by C. glutamicum, one might expect C. glutamicum is also an ideal host for the heterologous production of ectoine. In addition, C. glutamicum has no ectoine degradation pathway just like E. coli, capable of avoiding the consumption of ectoine and the formation of byproduct [64].

The lysine-producing strain C. glutamicum LYS-1 has been selected for the ectoine biosynthesis because it expresses a feedback inhibition resistant aspartokinase $\left(\mathrm{LysC}^{\mathrm{T} 311 \mathrm{I}}\right)$ and thus can synthesize large amount of ASA [65]. Becker et al. expressed the ectABCD gene 
cluster from Pseudomonas stutzeri in the genome of $C$. glutamicum LYS-1, with ectABCD under the control of a strong constitutive promoter tuf [66]. The $d d h$ gene coding for diaminopimelate dehydrogenase, which diverts most of the metabolic flux into the dehydrogenase branch of the lysine biosynthetic pathway when the ammonia level is high, was selected as the integration region to decrease the carbon flux from ASA to lysine. However, lysine still can be detected as a byproduct. To further prevent the excretion of lysine, the $l y s E$ gene encoding the lysine exporter LysE was knocked out. The deletion of lysE gene blocked the excretion of lysine but had no effect on the excretion of ectoine. Finally, the engineered C. glutamicum ECT-2 grown in low-salt medium $(0.03 \mathrm{M}$ salt $)$ accumulated $4.5 \mathrm{~g} / \mathrm{L}$ ectoine in a fed-batch fermentation, with a productivity of $0.28 \mathrm{~g} / \mathrm{L} / \mathrm{h}$. Given that only $0.4 \mathrm{~g} / \mathrm{L}$ hydroxyectoine was produced by C. glutamicum ECT-2 strain, the succinylase branch might divert a part of the carbon flux into by-product lysine, and the heterologous ectoine transporters might also affect the biosynthetic efficiency of ectoine in the engineered C. glutamicum.

In addition to expressing a previously described feedback inhibition resistant aspartokinase Lys $\mathrm{C}^{\mathrm{T} 311 \mathrm{I}}$, another lysine-producing strain C. glutamicum DM1729 has a variant pyruvate carboxylase $\mathrm{Pyc}^{\mathrm{P} 458 \mathrm{~S}}$ and a variant homoserine dehydrogenase $\mathrm{Hom}^{\mathrm{V} 59 \mathrm{~A}}$, which exhibits a higher activity than the wild-type pyruvate carboxylase Pyc and a lower activity than the wild-type homoserine dehydrogenase Hom, respectively. The enhanced activity of Pyc ${ }^{\text {P458S }}$ makes more oxaloacetate precursor available for lysine biosynthesis, while the impaired activity of Hom ${ }^{\mathrm{V} 59 \mathrm{~A}}$ limits the conversion of ASA to threonine and methionine [67]. C. glutamicum DM1729 could be a promising microbial chassis for enhanced production of ectoine since it has already achieved a good lysine production, which shares a lot of metabolic fluxes with ectoine biosynthesis. Therefore, Pérez-García et al. expressed the ectoine biosynthetic pathway in C. glutamicum DM1729 for the production of ectoine. The host genes $\operatorname{sugR}$ and $l d h A$-coding for a regulator and lactate dehydrogenase, respectively-were further deleted to improve the metabolism of glucose and avoid the formation of lactic acid [68]. The engineered strain C. glu-

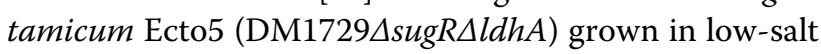
medium (0.01 M salt) accumulated $22 \mathrm{~g} / \mathrm{L}$ ectoine in a fed-batch fermentation, with a productivity of $0.32 \mathrm{~g} / \mathrm{L} / \mathrm{h}$ and a yield of $0.16 \mathrm{~g} / \mathrm{g}$ glucose, respectively. However, a large amount of by-product lysine still can be detected in the fermentation broth. Though attempt has been made to avoid the lysine efflux, the deletion of $l y s E$ gene results in dramatically decreased ectoine production and obviously impaired cell growth. Unlike C. glutamicum ECT-2, a further deletion of lysE in C. glutamicum Ecto5 may cause the metabolic imbalance of the bacterial cell.

More recently, Giesselmann et al. used C. glutamicum lys $C^{f b r}$ as starting strain for the heterologous production of ectoine [35]. A series of genetic modification on this lysine-producing strain was carried out to further improve the ectoine biosynthesis (Fig. 2b). Firstly, the lysE gene was deleted to block the excretion of lysine. Then, a transcription balance strategy was adopted to systematically optimize the ectoine biosynthetic pathway cloned from P. stutzeri, resulting in higher production levels of ectoine. Unlike the previous strategy of using one promoter to control the expression of ect $A B C$ genes, 19 synthetic promoters and 3 bicistronic linkers were employed to coordinate the coexpression of ect $A, e c t B$ and ect $C$ by creating an expression library with 185,193 variants. The engineered $C$. glutamicum ectABC ${ }^{\text {opt }}$ obtained by high-throughput screening of transformants produced $65 \mathrm{~g} / \mathrm{L}$ ectoine in a fed-batch fermentation fed with glucose-molasses medium (0.03 M salt), representing the highest ectoine titer to date. A good productivity of $1.16 \mathrm{~g} / \mathrm{L} / \mathrm{h}$ and a good yield of $0.19 \mathrm{~g} / \mathrm{g}$ glucose-molasses were also achieved in this fermentation process, with just a small amount of trehalose detected as by-product $(3 \mathrm{~g} / \mathrm{L})$. In addition, by comparing C. glutamicum ect $A B C^{\text {opt }}$ with a part of other mutants in the library, the expression ratio of ectA/ectB was found to be of great importance in determining the pathway flux, with the best ectoine producer exhibiting an EctA:EctB ratio of 1:3.1 by proteome analysis. This valuable finding facilitates the subsequent development of efficient chassis cells for further enhanced ectoine production.

\section{Hansenula polymorpha}

Hansenula polymorpha, which is a methylotrophic yeast, has been well developed for the expression of heterologous proteins, and it has also been used as a whole-cell biocatalyst [69-71]. In order to produce hydroxyectoine, synthetic ORFs encoding EctA, EctB, EctC and EctD from $H$. elongata were inserted into the genome of $H$. polymorpha. When grown in the medium supplemented with methanol and sorbitol as carbon source, the engineered $H$. polymorpha produced $2.8 \mathrm{~g} / \mathrm{L}$ hydroxyectoine, with only $0.044 \mathrm{~g} / \mathrm{L}$ ectoine as a by-product [72]. Moreover, almost no salt is required for efficient production of hydroxyectoine in $H$. polymorpha, exhibiting advantages over other microbial chassis in terms of low-salt production of hydroxyectoine. However, the long fermentation time $(144 \mathrm{~h})$ undoubtedly increases the cost of hydroxyectoine production, posing a problem to the industrial production of hydroxyectoine in $H$. polymorpha. 


\section{Microbial production of ectoine/hydroxyectoine based on next generation industrial biotechnology} With increasing stress from environmental pollution, growing attention is being paid to the microbial production of chemicals in a more environment-friendly manner. The "next generation industrial biotechnology" based on moderately halophilic bacteria has advantages over the current industrial biotechnology. Firstly, no strict sterilization is required for the media, decreasing the energy consumption and process complexity. Secondly, cheap plastic or even cement fermentation tanks can be used for mass production, avoiding the corrosion to the traditional steel fermentation tanks and reducing the production cost. Thirdly, sea water can be used directly with a simple processing, saving a lot of valuable fresh water.

Recently, Ma et al. developed an efficient de novo ectoine biosynthetic pathway by rational flux-tuning of the moderately halophilic Halomonas bluephagenesis in an open unsterile fed-batch fermentation (Fig. 3) [36]. They firstly confirmed the function of ect $A B C$ gene cluster from $H$. bluephagenesis by measuring the ectoine production in the start host $H$. bluephagenesis TD1.0 and the recombinant $E$. coli. Based on its native ectoine biosynthetic system, $H$. bluephagenesis TD1.0 grown in the medium supplemented with $1.03 \mathrm{M} \mathrm{NaCl}$ produced $0.63 \mathrm{~g} / \mathrm{L}$ ectoine for resisting the hyperosmotic stress. To circumvent host's regulatory system normally controlled by salt concentration and further enhance the production of ectoine, a T7-like plasmid expression system was employed to optimize the expression of ect$A B C$ in $H$. bluephagenesis TD1.0 and the doeA and ectD genes were deleted to prevent ectoine from degradation [73]. By adopting these strategies, up to $3.2 \mathrm{~g} / \mathrm{L}$ ectoine was obtained, representing a great improvement in ectoine production. Given chromosome integration of target genes enables the robust growth of chassis cells in antibiotic free medium, the ect $A B C$, lys $C$ and asd genes were inserted into the $H$. bluephagenesis chromosome for constitutive expression. In addition, the transcription levels of the three isolated gene clusters were finetuned using a GFP-based transcriptional tuning method. Finally, the engineered $H$. bluephagenesis TD-ADEL-58 produced $28 \mathrm{~g} / \mathrm{L}$ ectoine within $28 \mathrm{~h}$ in a fed-batch fermentation, with a concomitant production of poly- $\beta$ hydroxybutyrate (PHB) inside the cell as a co-product [36]. Therefore, the moderately halophilic $H$. bluephagenesis represents a promising chassis cell for co-production of ectoine and PHB, promoting the development of next generation industrial biotechnology.

\section{Conclusions}

Halomonas elongata is currently used as the major bacterium for the commercial production of ectoine. However, the high-salt fermentation broth used for efficient ectoine biosynthesis in this process is corrosive to the fermentation tank and difficult for the downstream wastewater treatment. Therefore, efforts have been made to develop other chassis cells for efficient production of ectoine/hydroxyectoine with lowered salt levels. The engineered model microorganisms such as $E$. coli and $C$. glutamicum are promising for the conventional industrial production of ectoine/hydroxyectoine, as

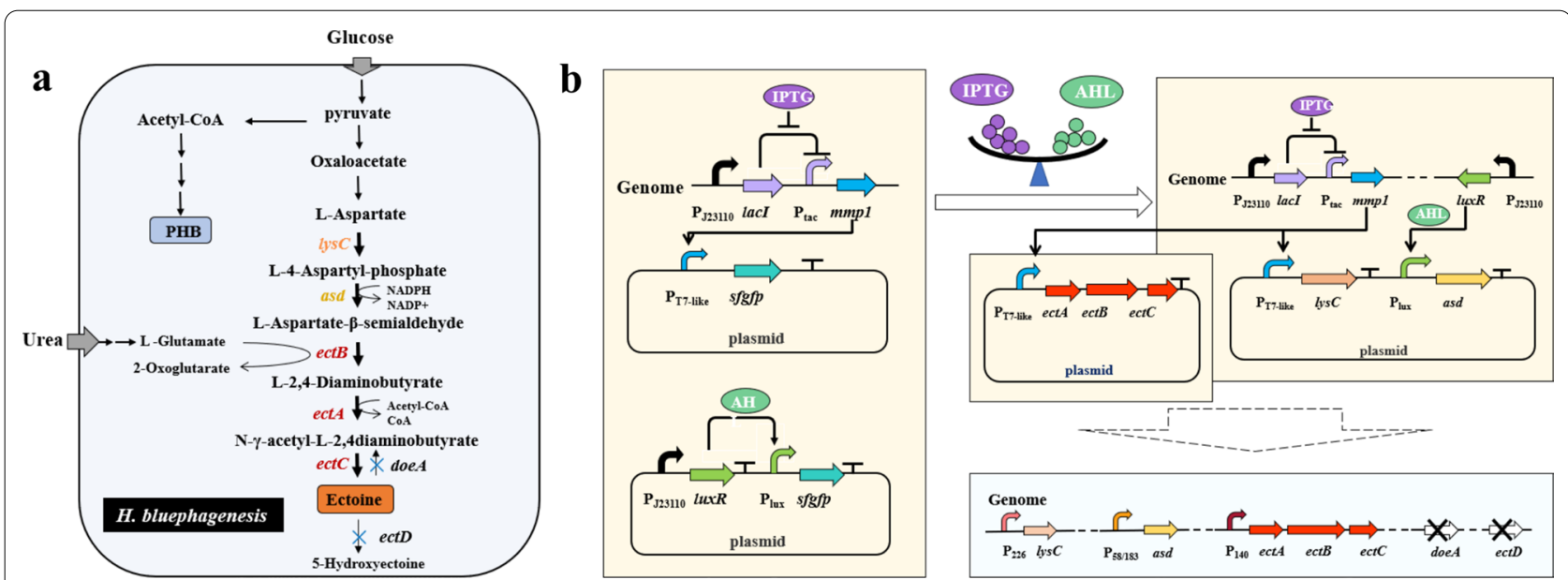

Fig. 3 Metabolic engineering modification strategy for ectoine and PHB co-production by H. bluephagenesis. a The reconstructed ectoine and PHB co-biosynthetic pathway in H. bluephagenesis. Modified metabolic fluxes of high efficiency involved in ectoine synthesis are shown in black bold lines. Genes doeA and ectD encoding ectoine hydrolase and ectoine hydroxylase, respectively, are deleted. $\mathbf{b}$ Application of two orthogonal inducible systems in ectoine synthesizes related genes. Two orthogonal inducible systems and fine-tuning of asd, lys $C$ and ect $A B C$ genes 
they are capable of producing large amount of ectoine/ hydroxyectoine under low salt conditions.

Given ASA is an important precursor molecule in ectoine/hydroxyectoine biosynthetic pathway, most of the model microorganisms employed for the heterologous production of ectoine/hydroxyectoine are good producers of aspartate-derived amino acids, which can provide ectoine/hydroxyectoine biosynthesis with sufficient ASA precursor. However, large amounts of amino acids are produced concomitantly as by-products. Though the disruption of the amino acid biosynthetic pathways is a preferred strategy for highspecificity production of ectoine/hydroxyectoine, the elimination of amino acid production usually results in the decreased production of ectoine/hydroxyectoine. Therefore, prevention of amino acid excretion could be considered as an alternative strategy to simplify the downstream processing of ectoine/hydroxyectoine.

Transcriptional balance has shown to be of great importance in ectoine production. High expression of Ect $B$ and high ratio of EctB/EctA are key to the efficient ectoine production, indicating that EctB is a rate-limiting enzyme in ectoine biosynthesis. With the fast development of multi-omics, molecular systems biology and synthetic biology, one might expect more economical production of ectoine and hydroxyectoine in future.

\begin{abstract}
Abbreviations
Ask: L-Aspartate kinase; Asd: L-Aspartate- $\beta$-semialdehyde dehydrogenase; EctB: L-2,4-Diaminobutyrate aminotransferase; EctA: L-2,4-Diaminobutyrate acetyltransferase; EctC: Ectoine synthase; EctD: Ectoine hydroxylase; EutABC: Hypothesized enzymes for hydroxyectoine degradation; DoeA: Ectoine hydrolase; DoeB: N-a-Acetyl-L-2,4-diaminobutyric acid deacetylase; DoeD: Diaminobutyric acid transaminase; DoeC: Aspartate-semialdehyde dehydrogenase; MSC: Mechanosensitive channels; ASA: Aspartate- $\beta$-semialdehyde; Pyc: Pyruvate carboxylase; Hom: Homoserine dehydrogenase.
\end{abstract}

\section{Acknowledgements}

The authors would like to thank all the members of Zheng Lab and Biobased Materials Center for their contributions on literature collection and critical reading of the manuscript.

\section{Authors' contributions}

$M L$ and $Y Z$ drafted the initial manuscript. $H L, M S, M J, L L$ and $Y Z$ revised the manuscript. All authors read and approved the final manuscript.

\section{Funding}

This work was supported by the National Key R\&D Program of China (2020YFA0906800), the National Natural Science Foundation of China (91851102 and 32070034) and the Senior User Project of RV KEXUE, Center for Ocean Mega-Science, Chinese Academy of Sciences (KEXUE2019GZ05).

\section{Availability of data and materials}

Not applicable.

\section{Declarations}

Ethics approval and consent to participate Not applicable.
Consent for publication

Not applicable.

\section{Competing interests}

The authors declare that they have no competing interests.

\section{Author details}

${ }^{1}$ State Key Laboratory of Microbial Resources, Institute of Microbiology, Chinese Academy of Sciences, No. 1 Beichen West Road, Chaoyang District, Beijing 100101, China. ${ }^{2}$ CAS Key Laboratory of Bio-Based Materials, Qingdao Institute of Bioenergy and Bioprocess Technology, Chinese Academy of Sciences, Qingdao, China. ${ }^{3}$ University of Chinese Academy of Sciences, Beijing, China. ${ }^{4}$ Department of Microbiology, University of Washington, Seattle, USA.

Received: 28 November 2020 Accepted: 16 March 2021

Published online: 26 March 2021

\section{References}

1. Rojas ER, Huang KC. Regulation of microbial growth by turgor pressure. Curr Opin Microbiol. 2018;42:62-70.

2. van den Berg J, Boersma AJ, Poolman B. Microorganisms maintain crowding homeostasis. Nat Rev Microbiol. 2017;15:309-18.

3. Kempf B, Bremer E. Uptake and synthesis of compatible solutes as microbial stress responses to high-osmolality environments. Arch Microbiol. 1998;170:319-30.

4. Brown AD. Microbial water stress. Bacteriol Rev. 1976;40:803-46.

5. Roberts MF. Organic compatible solutes of halotolerant and halophilic microorganisms. Saline Syst. 2005;1:5.

6. Becker J, Wittmann C. Microbial production of extremolytes-high-value active ingredients for nutrition, health care, and well-being. Curr Opin Biotechnol. 2020;65:118-28.

7. Galinski EA, Pfeiffer HP, Truper HG. 1,4,5,6-Tetrahydro-2-methyl-4-pyrimidinecarboxylic acid - a novel cyclic amino acid from halophilic phototrophic bacteria of the genus Ectothiorhodospira. Eur J Biochem. 1985;149:135-9.

8. Inbar L, Lapidot A. The structure and biosynthesis of new tetrahydropyrimidine derivatives in actinomycin D producer Streptomyces parvulus. Use of 13C- and 15N-labeled L-glutamate and 13C and 15N NMR spectroscopy. J Biol Chem. 1988;263:16014-22.

9. Pastor JM, Salvador M, Argandona M, Bernal V, Reina-Bueno M, Csonka LN Iborra JL, Vargas C, Nieto JJ, Canovas M. Ectoines in cell stress protection: uses and biotechnological production. Biotechnol Adv. 2010;28:782-801.

10. Graf R, Anzali S, Buenger J, Pfluecker F, Driller H. The multifunctional role of ectoine as a natural cell protectant. Clin Dermatol. 2008;26:326-33.

11. Buenger J, Driller H. Ectoine: an effective natural substance to prevent UVA-induced premature photoaging. Skin Pharmacol Physiol. 2004; 17:232-7.

12. Buenger J, Degwert J, Driller $\mathrm{H}$. The protective function of compatible solute ectoine on skin cells and its biomolecules with respect to UV-radiation, immunosuppression and membrane damage. IFSCC Mag. 2001:4:1-6.

13. Kanapathipillai M, Lentzen G, Sierks M, Park CB. Ectoine and hydroxyectoine inhibit aggregation and neurotoxicity of Alzheimer's beta-amyloid. FEBS Lett. 2005;579:4775-80.

14. Salapatek A, Bates M, Bilstein A, Patel D. Ectoin (R), a novel, non-drug, extremophile-based device, relieves allergic rhinoconjunctivitis symptoms in patients in an environmental exposure chamber model. J Allergy Clin Immunol. 2011;127:AB202.

15. Strong PJ, Kalyuzhnaya M, Silverman J, Clarke WP. A methanotroph-based biorefinery: potential scenarios for generating multiple products from a single fermentation. Bioresour Technol. 2016;215:314-23.

16. Tanne C, Golovina EA, Hoekstra FA, Meffert A, Galinski EA. Glass-forming property of hydroxyectoine is the cause of its superior function as a desiccation protectant. Front Microbiol. 2014;5:150.

17. Garcia-Estepa R, Argandona M, Reina-Bueno M, Capote N, IglesiasGuerra F, Nieto JJ, Vargas C. The ectD gene, which is involved in the synthesis of the compatible solute hydroxyectoine, is essential for thermoprotection of the halophilic bacterium Chromohalobacter salexigens. J Bacteriol. 2006;188:3774-84. 
18. Peters P, Galinski EA, Truper HG. The biosynthesis of ectoine. FEMS Microbiol Lett. 1990;71:157-62.

19. Ono H, Sawada K, Khunajakr N, Tao T, Yamamoto M, Hiramoto M, Shinmyo A, Takano M, Murooka Y. Characterization of biosynthetic enzymes for ectoine as a compatible solute in a moderately halophilic eubacterium, Halomonas elongata. J Bacteriol. 1999;181:91-9.

20. Schiraldi C, Maresca C, Catapano A, Galinski EA, De Rosa M. Highyield cultivation of Marinococcus M52 for production and recovery of hydroxyectoine. Res Microbiol. 2006;157:693-9.

21. Bursy J, Pierik AJ, Pica N, Bremer E. Osmotically induced synthesis of the compatible solute hydroxyectoine is mediated by an evolutionarily conserved ectoine hydroxylase. J Biol Chem. 2007;282:31147-55.

22. Calderon MI, Vargas C, Rojo F, Iglesias-Guerra F, Csonka LN, Ventosa A, Nieto JJ. Complex regulation of the synthesis of the compatible solute ectoine in the halophilic bacterium Chromohalobacter salexigens DSM 3043. Microbiology. 2004;150:3051-63.

23. Kol S, Merlo ME, Scheltema RA, de Vries M, Vonk RJ, Kikkert NA, Dijkhuizen L, Breitling R, Takano E. Metabolomic characterization of the salt stress response in Streptomyces coelicolor. Appl Environ Microbiol. 2010;76:2574-81

24. Onraedt A, De Muynck C, Walcarius B, Soetaert W, Vandamme E. Ectoine accumulation in Brevibacterium epidermis. Biotechnol Lett. 2004;26:1481-5.

25. Vargas C, Jebbar M, Carrasco R, Blanco C, Calderon MI, Iglesias-Guerra F, Nieto JJ. Ectoines as compatible solutes and carbon and energy sources for the halophilic bacterium Chromohalobacter salexigens. J Appl Microbiol. 2006;100:98-107.

26. Schwibbert K, Marin-Sanguino A, Bagyan I, Heidrich G, Lentzen G, Seitz H, Rampp M, Schuster SC, Klenk H-P, Pfeiffer F, et al. A blueprint of ectoine metabolism from the genome of the industrial producer Halomonas elongata DSM 2581 (T). Environ Microbiol. 2011;13:1973-94.

27. Schulz A, Stoeveken N, Binzen IM, Hoffmann T, Heider J, Bremer E. Feeding on compatible solutes: a substrate-induced pathway for uptake and catabolism of ectoines and its genetic control by EnuR. Environ Microbiol. 2017;19:926-46.

28. Mais C-N, Hermann L, Altegoer F, Seubert A, Richter AA, Wernersbach I, Czech L, Bremer E, Bange G. Degradation of the microbial stress protectants and chemical chaperones ectoine and hydroxyectoine by a bacterial hydrolase-deacetylase complex. J Biol Chem. 2020;295:9087-104.

29. Kunte HJ, Lentzen G, Galinski EA. Industrial production of the cell protectant ectoine: protection mechanisms, processes, and products. Curr Biotechnol. 2014;3:10-25.

30. Sauer T, Galinski EA. Bacterial milking: a novel bioprocess for production of compatible solutes. Biotechnol Bioeng. 1998;57:306-13.

31. Onraedt AE, Walcarius BA, Soetaert WK, Vandamme EJ. Optimization of ectoine synthesis through fed-batch fermentation of Brevibacterium epidermis. Biotechnol Prog. 2005;21:1206-12.

32. Schubert T, Maskow T, Benndorf $D$, Harms H, Breuer U. Continuous synthesis and excretion of the compatible solute ectoine by a transgenic, nonhalophilic bacterium. Appl Environ Microbiol. 2007;73:3343-7.

33. Lang Y-J, Bai L, Ren Y-N, Zhang L-H, Nagata S. Production of ectoine through a combined process that uses both growing and resting cells of Halomonas salina DSM 5928(T). Extremophiles. 2011;15:303-10.

34. Ning $Y$, Wu $X$, Zhang $C, X u Q$, Chen $N$, Xie X. Pathway construction and metabolic engineering for fermentative production of ectoine in Escherichia coli. Metab Eng. 2016;36:10-8.

35. Giesselmann G, Dietrich D, Jungmann L, Kohlstedt M, Jeon EJ, Yim SS, Sommer F, Zimmer D, Muehlhaus T, Schroda M, et al. Metabolic engineering of Corynebacterium glutamicum for high-level ectoine production: design, combinatorial assembly, and implementation of a transcriptionally balanced heterologous ectoine pathway. Biotechnol J. 2019;14:1800417.

36. Ma H, Zhao Y-Q, Huang W-Z, Zhang L-Z, Wu F-Q, Ye J-W, Chen G-Q. Rational flux-tuning of Halomonas bluephagenesis for co-production of bioplastic PHB and ectoine. Nat Commun. 2020;11:12.

37. Cox CD, Bavi N, Martinac B. Bacterial mechanosensors. Annu Rev Physiol. 2018;80:71-93.

38. Vandrich J, Pfeiffer F, Alfaro-Espinoza G, Kunte HJ. Contribution of mechanosensitive channels to osmoadaptation and ectoine excretion in Halomonas elongata. Extremophiles. 2020;24:421-32.
39. Grammann K, Volke A, Kunte HJ. New type of osmoregulated solute transporter identified in halophilic members of the Bacteria domain: TRAP transporter TeaABC mediates uptake of ectoine and hydroxyectoine in Halomonas elongata DSM 2581(T). J Bacteriol. 2002;184:3078-85.

40. Prabhu J, Schauwecker F, Grammel N, Keller U, Bernhardt M. Functional expression of the ectoine hydroxylase gene (thpD) from Streptomyces chrysomallus in Halomonas elongata. Appl Environ Microbiol. 2004;70:3130-2.

41. Zhang $L-H$, Lang Y-J, Nagata S. Efficient production of ectoine using ectoine-excreting strain. Extremophiles. 2009;13:717-24.

42. Chen W-C, Hsu C-C, Lan JC-W, Chang Y-K, Wang L-F, Wei Y-H. Production and characterization of ectoine using a moderately halophilic strain Halomonas salina. J Biosci Bioeng. 2018;125:578-84.

43. Chen W-C, Hsu C-C, Wang L-F, Lan JCW, Chang Y-K, Wei Y-H. Exploring useful fermentation strategies for the production of hydroxyectoine with a halophilic strain, Halomonas salina BCRC 17875. J Biosci Bioeng. 2019;128:332-6.

44. Yang C-Y, Wang Z, Li Y, Niu Y, Du M-F, He X-F, Ma C-Q, Tang H-Z, Xu P. Metabolic versatility of halotolerant and alkaliphilic strains of Halomonas isolated from alkaline black liquor. Bioresour Technol. 2010;101:6778-84.

45. Zhao Q, Meng Y-M, Li S-N, Lv P-W, Xu P, Yang C-Y. Genome sequence of Halomonas hydrothermalis $Y 2$, an efficient ectoine-producer isolated from pulp mill wastewater. J Biotechnol. 2018;285:38-41.

46. Zhao Q, Li S-N, Lv P-W, Sun S-M, Ma C-Q, Xu P, Su H-J, Yang C-Y. High ectoine production by an engineered Halomonas hydrothermalis $\mathrm{Y} 2$ in a reduced salinity medium. Microb Cell Fact. 2019;18:1-12.

47. Cheng B, Meng Y-W, Cui Y-B, Li C-F, Tao F, Yin H-J, Yang C-Y, Xu P. Alkaline response of a halotolerant alkaliphilic Halomonas strain and functional diversity of its $\mathrm{Na}^{+}\left(\mathrm{K}^{+}\right) / \mathrm{H}^{+}$antiporters. J Biol Chem. 2016;291:26056-65.

48. Baneyx F. Recombinant protein expression in Escherichia coli. Curr Opin Biotechnol. 1999:10:411-21.

49. Jebbar M, Talibart R, Gloux K, Bernard T, Blanco C. Osmoprotection of Escherichia coli by ectoine: uptake and accumulation characteristics. J Bacteriol. 1992;174:5027-35.

50. Louis $\mathrm{P}$, Galinski EA. Characterization of genes for the biosynthesis of the compatible solute ectoine from Marinococcus halophilus and osmoregulated expression in Escherichia coli. Microbiology. 1997;143:1141-9.

51. He Y-Z, Gong J, Yu H-Y, Tao Y, Zhang S, Dong Z-Y. High production of ectoine from aspartate and glycerol by use of whole-cell biocatalysis in recombinant Escherichia coli. Microb Cell Fact. 2015:14:1-10.

52. Lee KH, Park JH, Kim TY, Kim HU, Lee SY. Systems metabolic engineering of Escherichia coli for L-threonine production. Mol Syst Biol. 2007:3:149.

53. Song CW, Lee J, Ko Y-S, Lee SY. Metabolic engineering of Escherichia coli for the production of 3-aminopropionic acid. Metab Eng. 2015;30:121-9.

54. Chen W, Zhang S, Jiang P-X, Yao J, He Y-Z, Chen L-C, Gui X-W, Dong Z-Y, Tang S-Y. Design of an ectoine-responsive AraC mutant and its application in metabolic engineering of ectoine biosynthesis. Metab Eng. 2015;30:149-55.

55. Hillier HT, Altermark B, Leiros I. The crystal structure of the tetrameric DABA-aminotransferase EctB, a rate-limiting enzyme in the ectoine biosynthesis pathway. FEBS J. 2020;287(21):4641-58.

56. Seip B, Galinski EA, Kurz M. Natural and engineered hydroxyectoine production based on the Pseudomonas stutzeriectABCD-ask gene cluster. Appl Environ Microbiol. 2011;77:1368-74.

57. Czech L, Stoeveken N, Bremer E. EctD-mediated biotransformation of the chemical chaperone ectoine into hydroxyectoine and its mechanosensitive channel-independent excretion. Microb Cell Fact. 2016;15:1-16.

58. Dinnibier U, Limpinsel E, Schmid R, Bakker EP. Transient accumulation of potassium glutamate and its replacement by trehalose during adaptation of growing cells of Escherichia coli K-12 to elevated sodium chloride concentrations. Arch Microbiol. 1988;150:348-57.

59. Bethlehem L, Moritz KD. Boosting Escherichia coli's heterologous production rate of ectoines by exploiting the non-halophilic gene cluster from Acidiphilium cryptum. Extremophiles. 2020;24:733-47.

60. Becker J, Wittmann C. Bio-based production of chemicals, materials and fuels-Corynebacterium glutamicum as versatile cell factory. Curr Opin Biotechnol. 2012;23:631-40.

61. Wendisch VF, Jorge JMP, Perez-Garcia F, Sgobba E. Updates on industrial production of amino acids using Corynebacterium glutamicum. World J Microbiol Biotechnol. 2016;32:105. 
62. Eggeling L, Bott M. A giant market and a powerful metabolism: L-lysine provided by Corynebacterium glutamicum. Appl Microbiol Biotechnol. 2015;99:3387-94.

63. Wittmann C, Becker J. The L-lysine story: from metabolic pathways to industrial production. In: Microbiology monographs. Berlin: Springer; 2007. p. 39-70.

64. Kalinowski J, Bathe B, Bartels D, Bischoff N, Bott M, Burkovski A, Dusch N, Eggeling L, Eikmanns BJ, Gaigalat L, et al. The complete Corynebacterium glutamicum ATCC 13032 genome sequence and its impact on the production of L-aspartate-derived amino acids and vitamins. J Biotechnol. 2003;104:5-25.

65. Becker J, Zelder O, Haefner S, Schroeder H, Wittmann C. From zero to hero-design-based systems metabolic engineering of Corynebacterium glutamicum for L-lysine production. Metab Eng. 2011;13:159-68.

66. Becker J, Schaefer R, Kohlstedt M, Harder BJ, Borchert NS, Stoeveken N, Bremer E, Wittmann C. Systems metabolic engineering of Corynebacterium glutamicum for production of the chemical chaperone ectoine. Microb Cell Fact. 2013;12:1-16.

67. Georgi T, Rittmann D, Wendisch VF. Lysine and glutamate production by Corynebacterium glutamicum on glucose, fructose and sucrose: roles of malic enzyme and fructose-1,6-bisphosphatase. Metab Eng. 2005;7:291-301.

68. Perez-Garcia F, Ziert C, Risse JM, Wendisch VF. Improved fermentative production of the compatible solute ectoine by Corynebacterium glutamicum from glucose and alternative carbon sources. J Biotechnol. 2017;258:59-68
69. Eilert E, Hollenberg CP, Piontek M, Suckow M. The use of highly expressed FTH1 as carrier protein for cytosolic targeting in Hansenula polymorpha. J Biotechnol. 2012;159:172-6.

70. Mayer AF, Hellmuth K, Schlieker H, Lopez-Ulibarri R, Oertel S, Dahlems U, Strasser AWM, van Loon A. An expression system matures: a highly efficient and cost-effective process for phytase production by recombinant strains of Hansenula polymorpha. Biotechnol Bioeng. 1999;63:373-81.

71. Gellissen G, Piontek M, Dahlems U, Jenzelewski V, Gavagan JE, DiCosimo R, Anton DL, Janowicz ZA. Recombinant Hansenula polymorpha as a biocatalyst: coexpression of the spinach glycolate oxidase (GO) and the S-cerevisiae catalase T (CTT $)$ gene. Appl Microbiol Biotechnol. 1996;46:46-54.

72. Eilert E, Kranz A, Hollenberg CP, Piontek M, Suckow M. Synthesis and release of the bacterial compatible solute 5-hydroxyectoine in Hansenula polymorpha. J Biotechnol. 2013;167:85-93.

73. Zhao H, Zhang HMQ, Chen X-B, Li T, Wu Q, Ouyang Q, Chen G-Q. Novel T7-like expression systems used for Halomonas. Metab Eng. 2017;39:128-40.

\section{Publisher's Note}

Springer Nature remains neutral with regard to jurisdictional claims in published maps and institutional affiliations.
Ready to submit your research? Choose BMC and benefit from:

- fast, convenient online submission

- thorough peer review by experienced researchers in your field

- rapid publication on acceptance

- support for research data, including large and complex data types

- gold Open Access which fosters wider collaboration and increased citations

- maximum visibility for your research: over $100 \mathrm{M}$ website views per year

At BMC, research is always in progress.

Learn more biomedcentral.com/submissions 